\title{
Manufacturing of Dexamethasone-Poly (D,L-Lactide-co-Glycolide) Implants Using Hot-Melt Extrusion: Within- and Between-Batch Product Performance Comparisons
}

\author{
Ryan A. Kelley, ${ }^{1}$ Alireza Ghaffari, ${ }^{1, *}$ Yan Wang, ${ }^{2}$ Stephanie Choi, ${ }^{2}$ Jonathan R. Taylor, \\ Rachel R. Hartman, ${ }^{1}$ and Uday B. Kompella ${ }^{1}$
}

\begin{abstract}
Purpose: Reliable drug therapy with injectable intravitreal implants requires implants of consistent quality. The purpose of this study was to prepare dexamethasone-poly(D,L-lactide-co-glycolide) (PLGA) biodegradable implants and assess implant quality within and between batches for different polymer compositions.

Methods: Implants containing $20 \% \mathrm{w} / \mathrm{w}$ dexamethasone with 3 theoretical rates of release (fast, intermediate, and slow) were manufactured with decreasing proportion of acid-terminated PLGA (50:50) and increasing proportion of ester-terminated PLGA (50:50) in a batch process using hot-melt extrusion. The implants were manufactured without and with in-process modification of extrusion/conveyor speed in the late phase of each batch. Implant samples collected at early, middle, and late phases of each batch were analyzed for diameter, drug loading, mechanical properties (strength and toughness), and drug release.

Results: With a fixed process, unlike a modified process with an increase in extrusion speed and reduction of conveyor speed in the late phase, all implant formulations tended to decrease in diameter and mechanical properties in the late phase. Drug release profiles for the intermediate and slow release compositions were similar with or without process modification, unlike the fast release composition. Addition of ester-terminated PLGA resulted in a slower drug release. When all formulations are grouped together, the implant diameter exhibited a moderate correlation with mechanical properties, but no correlation was observed with drug release. Conclusions: Within a hot-melt extrusion batch process, the dexamethasone-PLGA implant diameter and hence toughness and strength tend to decline in the latter phase. In-process adjustment of extrusion and conveyor speeds can improve batch consistency and, potentially, implant integrity or performance during or after injection. Process changes did not affect drug release for 2 of the 3 implant compositions.
\end{abstract}

Keywords: dexamethasone, intravitreal implant, hot-melt extrusion, ocular drug delivery, biodegradable implant

\section{Introduction}

D EXAMETHASONE IS a corticosteroid with potent glucocorticoid receptor agonist activity that can be injected into the vitreous to treat inflammation in diseases affecting the back of the eye. Experimentally, injections of corticosteroids have been shown to reduce vascular endothelial growth factor expression, downregulate inflammatory adhesion molecules, downregulate leukocyte migration, re- duce vascular leakage, and reduce prostaglandin levels. ${ }^{1-8}$ Ocular administration of corticosteroids dates back to 1974 when Graham and Peyman used dexamethasone to treat an induced model of infectious endophthalmitis. ${ }^{9}$ By the 2000s, the corticosteroid, triamcinolone, delivered through intravitreal injections was being used to treat a wide variety of ocular diseases. ${ }^{10-16}$ While corticosteroids, triamcinolone, fluocinolone, and dexamethasone, are all currently approved to treat ocular inflammation, dexamethasone and fluocinolone

\footnotetext{
${ }^{1}$ Skaggs School of Pharmacy and Pharmaceutical Sciences, University of Colorado Anschutz Medical Campus, Aurora, Colorado, USA.

${ }^{2}$ Food and Drug Administration, White Oak Campus, Silver Spring, Maryland.

*Current affiliation: NextHerbal Labs, LLC, Englewood, Colorado, USA.
} 
are 5 times more potent than triamcinolone. ${ }^{17,18}$ Unfortunately, corticosteroids have a short half-life of a few hours in the vitreous humor of the eye. ${ }^{19,20}$ Thus, a corticosteroid in solution would require numerous injections to achieve therapeutic benefit. To overcome this limitation in ocular treatment, a sustained release dexamethasone implant has been developed. Dexamethasone implants $(0.7 \mathrm{mg}$ of dexamethasone, Ozurdex ${ }^{\circledR}$, Allergan, Inc., Irvine, CA) are currently approved by the FDA to treat diabetic macular edema, macular edema due to branch and central retinal vein occlusions, and noninfectious posterior segment uveitis. ${ }^{21}$ These implants consist of a mixture of dexamethasone and poly(D,L-lactide-co-glycolide) (PLGA); however, the type of PLGA used is not mentioned in the product prescribing information. The implant is injected into the vitreous using a 22-gauge applicator that injects the implant through the pars plana into the vitreous humor.

Following intravitreal injection of $0.7 \mathrm{mg}$ of dexamethasone containing PLGA sustained release implants, Chang-Lin et al. ${ }^{22}$ found that dexamethasone was present in the vitreous and retina of rhesus macaques for up to 6 months. At 2 months, peak concentrations (Cmax) in the retina and vitreous were $1,110 \pm 284 \mathrm{ng} / \mathrm{g}$ and $213 \pm 49 \mathrm{ng} / \mathrm{mL}$, respectively, while over the course of 6 months, the area under the curves (AUCs) in the retina and vitreous were 47,200 ng.days/g and 11,300 ng.days/mL, respectively. ${ }^{22}$ Comparatively, ChangLin et al. ${ }^{23}$ found that in rabbit eyes at 22 days, the Cmax in vitreous humor was $791 \mathrm{ng} / \mathrm{mL}$, with a corresponding AUC of $13,600 \mathrm{ng}$.days/mL over 31 days. Similarly, at 15 days, the Cmax in retina was $4,110 \mathrm{ng} / \mathrm{g}$, with a corresponding AUC of 67,600 ng.days/g over 31 days. $^{23}$ While most of the dexamethasone from commercial implants is released during the first 8 weeks, the release continues beyond this time at a lower level and drug effects are expected to persist for up to 6 months. ${ }^{22-24}$

Dexamethasone-PLGA implants can be prepared by hotmelt extrusion, a batch process that mixes materials between 2 rotating screws inside a barrel while being heated above the PLGA glass transition temperature and continuously extruded through a die of a particular diameter until the material is exhausted. This extrudate is fed onto a conveyor belt where it rapidly solidifies at room temperature. However, the speed of the conveyor belt must also be taken into account to obtain implants of desired diameters. High speeds might result in smaller diameters and vice versa. As the barrel begins to lose material, the rate of volume extruded is expected to decrease. This might affect implant quality as the conveyor speed does not compensate. Therefore, the purpose of this study was to determine the reproducibility of implant quality and performance parameters, including drug loading, diameter, mechanical properties, and release, within and across batches for dexamethasone-PLGA implants. We studied these parameters throughout the extrusion process, specifically at the beginning, middle, and end phases of each batch, to understand how the extrusion process affects implant properties. We assessed 3 implant compositions, fast, intermediate, and slow release, based on decreasing theoretical degradation rates of the polymer mixes containing PLGA 50:50 polymer with acid or ester end groups. While the theoretical fast release composition employed the highest content of PLGA 50:50 acid end group polymer, the slow release composition employed the highest content of PLGA 50:50 ester end group polymer since the ester end group polymer is known to degrade more slowly when compared with the acid end group polymer. ${ }^{25}$

\section{Methods}

\section{Materials and formulations}

Dexamethasone (D4902; Shanxi Jinjin Chemical Co. Ltd., Hejin, China; about $2 \mu \mathrm{m}$ in particle size) was mixed with either 50:50 PLGA/acid-terminated or 50:50 PLGA/ ester-terminated polymer (PLGA) (B6013-1/B6017-1; Durect Corp., Birmingham, AL). The PLGA polymers used in this study, while being similar in lactic: glycolic acid ratio and inherent viscosity, differed in their end capping. While 1 polymer has acid end groups, the other has ester end groups. Prior studies demonstrated that the PLGA polymer with acid end groups degrades more rapidly due to its hydrophilic nature when compared with the polymer with ester end groups. ${ }^{25}$ By varying the ester end group polymer content, 3 formulations were prepared with the intent of imparting fast $(\mathrm{F} 1)$, intermediate $(\mathrm{F} 2)$, and slow $(\mathrm{F} 3)$ degradation rates and, as a consequence, different release rates for the 3 implant formulations. Three implant formulations were prepared as per the composition shown in Table 1. For each formulation, a total of $10 \mathrm{~g}$ of the mixture was ground into a fine powder using a pestle and mortar.

\section{Implant manufacture}

All implants were prepared by hot-melt extrusion using a HAAKE MiniCTW (Thermo Fisher, Waltham, MA). The temperature was set to $100^{\circ} \mathrm{C}$ and allowed to reach this set point. A $0.3 \mathrm{~mm}$ diameter orifice die plate was attached to the barrel chamber. The torque was set to $0.2 \mathrm{Nm}$. Then, $10 \mathrm{~g}$ of dexamethasone-PLGA material was then slowly fed into the feeding cylinder and into the barrel for mixing. The batch was allowed to mix for $10 \mathrm{~min}$. Following mixing, the screw (extrusion) speed was set to $20 \mathrm{rpm}$ and the material was extruded onto an M22 transport conveyor at a speed setting of 3.5 (Profilex Systems, S.A., Troisvierges, Luxembourg). In the case of implants produced without conveyor adjustment (fixed process; batch 1), implants were extruded at $20 \mathrm{rpm}$ and a conveyor speed of 3.5 throughout the entire extrusion process. In separate batches (B2 and B3; modified process), the conveyor was set to 3.5 initially and

Table 1. Formulations Used to Create Dexamethasone/Poly(D,L-Lactide-Co-Glycolide) Implants

\begin{tabular}{llcrr}
\hline Formulation & Release & Dexamethasone & PLGA (acid terminated) & PLGA (ester terminated) \\
\hline 1 & Fast & $20 \%(2.0 \mathrm{~g})$ & $80 \%(8.0 \mathrm{~g})$ & $0 \%(0.0 \mathrm{~g})$ \\
2 & Intermediate & $20 \%(2.0 \mathrm{~g})$ & $64 \%(6.4 \mathrm{~g})$ & $16 \%(1.6 \mathrm{~g})$ \\
3 & Slow & $20 \%(2.0 \mathrm{~g})$ & $48 \%(4.8 \mathrm{~g})$ & $32 \%(3.2 \mathrm{~g})$ \\
\hline
\end{tabular}

PLGA, poly(D,L-lactide-co-glycolide). 
then reduced to 3 at 42 min when the implant diameter fell well below $0.40 \mathrm{~mm}$, while the extrusion speed was increased to $30 \mathrm{rpm}$. At $49 \mathrm{~min}$, the extrusion speed was increased to $40 \mathrm{rpm}$. At $53 \mathrm{~min}$, the conveyor speed was reduced to a speed setting of 2.5 , while the extrusion speed was increased to $60 \mathrm{rpm}$. At $60 \mathrm{~min}$, when there was not enough material left to extrude, manufacturing was terminated. This control of extrusion speed and conveyor speed allowed the late-phase production of implants with diameters of about $0.40 \mathrm{~mm}$. A total of 115 strands, $\sim 11^{\prime \prime}$ in length, were collected through the extrusion process. Approximately strands 1 through 15 were labeled as early extrusions, strands 55-69 were labeled as middle extrusions, and strands 100-114 were labeled as late extrusions. Early, middle, and late extrusions were stored separately in a vacuum chamber at room temperature and protected from light. Hot-melt extrusion parameters are shown in Table 2 .

\section{Mechanical property measurement}

Implant strands were cut to 10 -mm lengths and measured using a Mitutoyo blade micrometer (156-101-10; Takatsu$\mathrm{ku}$, Kawasaki, Japan), and the diameters were recorded. Implant strength and toughness were measured using a TA.XTplus texture analyzer (Stable Micro Systems Ltd., Surrey, United Kingdom) and values were obtained utilizing the exponent software (v6,1,9,0) and the following typical conditions. Implants were placed at equal distances on the TA-92-M fixed in a TA-90 stage (Stable Micro Systems Ltd.), with a gap distance of $5 \mathrm{~mm}$. Implants were cut with a 20-mm blade apparatus under the test mode "compression." Pretest speed and test speed were set to $0.1 \mathrm{~mm} / \mathrm{s}$ with a post-test speed of $10 \mathrm{~mm} / \mathrm{s}$ to reset the blade height. Target mode was set to a distance of $1 \mathrm{~mm}$ and trigger type was set to pretravel at a distance of $0.25 \mathrm{~mm}$. Strain was kept at $10 \%$ throughout the measurements with a trigger force of $5 \mathrm{~g}$ and a break sensitivity of $10 \mathrm{~g}$. The blade was placed directly above the implant, but not touching it, and the program was initiated. Implant strength was measured as the maximum force $(\mathrm{g})$ required to break the implant, and implant toughness was measured as the maximum force versus time curve area $(\mathrm{g} \times \mathrm{s})$. Statistical analysis was completed using a 2-tailed Student's $t$-test.

\section{Dexamethasone quantification using spectrophotometry}

A standard calibration curve of dexamethasone was obtained with a SpectraMax M5 (Molecular devices,
Sunnyvale, CA) spectrophotometer and the SoftMax Pro (v5) software at an absorbance wavelength of $260 \mathrm{~nm}$. Dexamethasone absorbance was measured in Corning ${ }^{\circledR} \mathrm{UV}$ transparent 96-well plates (CL S363; Sigma-Aldrich, St. Louis, MO).

\section{Drug loading}

For drug loading determination, about 10-mm implant lengths were cut and weighed to be around $1-2 \mathrm{mg}$. Implants were then completely dissolved in $1 \mathrm{~mL}$ of dimethyl sulfoxide (DMSO). Implants were measured for dexamethasone content using spectrophotometry at $260 \mathrm{~nm}$. Standard curves were generated for each implant type relative to PLGA and dexamethasone controls. All stocks were completely dissolved in $1 \mathrm{~mL}$ of DMSO. A standard curve was generated in the range of $6.25-500 \mu \mathrm{g} / \mathrm{mL}$ dexamethasone. Percent loading was calculated by dividing the amount in the sample with the theoretical loading and then multiplying by 100 . Two data points with no detectable drug were considered as outliers. Statistical analysis was completed using a multifactorial analysis of variance.

\section{In vitro release}

Individual implants were weighed out to $\sim 1-2 \mathrm{mg}$ each and exact weights recorded. Implants were then submerged in $5 \mathrm{~mL}$ of $1 \times$ phosphate-buffered saline (PBS). These parameters were chosen because the aqueous solubility of dexamethasone is $0.1 \mathrm{mg} / \mathrm{mL}$. Theoretically, 200$400 \mu \mathrm{g}$ of implant weight is dexamethasone. Therefore, $5 \mathrm{~mL}$ provides a 1.5-2.5-fold required volume for complete dissolution of the drug. Solutions containing dexamethasone implants were incubated in 5-mL HPLC vials at $37^{\circ} \mathrm{C}$ in a MaxQ 4000 benchtop orbital shaker (Thermo Fisher Scientific, Waltham, MA) set to $150 \mathrm{rpm}$. Five hundred-microliter samples were collected and stored at $-80^{\circ} \mathrm{C}$ until the time of analysis, and $500 \mu \mathrm{L}$ of fresh $1 \times$ PBS (stored in the incubator with samples) was added back to the sample solution. For fast, intermediate, and slow release implants, samples were removed at 1, 2, 3, 4, 5, 6, and 7 weeks. All samples were thawed to room temperature and analyzed for dexamethasone concentration by spectrophotometry at $260 \mathrm{~nm}$ in UV-transparent 96-well plates using SpectraMax M5. Percent dexamethasone release was calculated by quantification of absorbance at $260 \mathrm{~nm}$ according to the standard curve of pure dexamethasone in solution and the theoretical drug loading. Calculations were adjusted to account for the dilution factor of sampling volume.

Table 2. DeXamethasone/Poly(D,L-Lactide-co-Glycolide) Hot-Melt Extrusion Parameters

\begin{tabular}{|c|c|c|c|c|c|c|c|}
\hline Rotor control & $\begin{array}{c}\text { Temperature } \\
\left({ }^{\circ} \mathrm{C}\right)\end{array}$ & $\begin{array}{c}\text { Mixing } \\
\text { torque }(\mathrm{Nm})\end{array}$ & $\begin{array}{c}\text { Mixing } \\
\text { time (min) }\end{array}$ & $\begin{array}{c}\text { Die plate } \\
\text { diameter }(\mathrm{mm})\end{array}$ & Extrusion speed & Rotor speed & $\begin{array}{l}\text { Extrusion } \\
\text { time (min) }\end{array}$ \\
\hline $\begin{array}{l}\text { Without rotor } \\
\text { control }\end{array}$ & 100 & 0.2 & 10 & 0.3 & $20 \mathrm{rpm}(60 \mathrm{~min})$ & $3.5(60 \mathrm{~min})$ & 60 \\
\hline $\begin{array}{l}\text { With rotor } \\
\text { control }\end{array}$ & 100 & 0.2 & 10 & 0.3 & $\begin{array}{l}20 \mathrm{rpm}(42 \mathrm{~min}), \\
30 \mathrm{rpm}(7 \mathrm{~min}), \\
40 \mathrm{rpm}(4 \mathrm{~min}), \\
60 \mathrm{rpm}(7 \mathrm{~min})\end{array}$ & $\begin{array}{l}3.5(42 \mathrm{~min}), \\
3.0(11 \mathrm{~min}), \\
2.5(7 \mathrm{~min})\end{array}$ & 60 \\
\hline
\end{tabular}




\section{Similarity of the in vitro release profiles of batches produced with and without process modification}

To determine if the release profiles of batches produced with process modification in the late phase (batches 2 and 3 ) were similar to the profiles of the batch produced with the fixed process (batch 1), the method described by Martinez and Zhao was used. ${ }^{26}$ Using the release profile of batch 1 as a reference ( $n=9$, combining early, middle, and late phases), tolerance limits (TLs) were estimated for each time point with $95 \%$ confidence. TLs define the predicted limits within which 99\% of the batch 1 release values will fall at each time point. The release profiles of both batches 2 and 3 were then plotted with TLs, TL $\pm 5 \%$, and TL $\pm 15 \%$. The release profiles of batches with the modified process were determined to be similar to fixed process batches if all release values fell within TL $\pm 15 \%$. Statistical analyses were performed, and graphics were generated using $\mathrm{R}$ (version 3.6.2).

\section{Results}

\section{Implant diameters decreased during extrusion}

Batch 1 (B1) of each formulation used the batch process without late-phase modification of extrusion speed or conveyor belt speed. Batches 2 and 3 (B2 and B3) of each formulation are batches with a modification of the above process parameters during the late phase. Early, middle, and late extrusions were cut to $10-\mathrm{mm}$ implants and the diameter was measured using a blade micrometer (Fig. 1). For all B1 formulations, the implant diameters were below $0.40 \mathrm{~mm}$ in the late extrusion phase, compared with the intended batch diameter of about $0.4 \mathrm{~mm}$ (Fig. 1). It was then hypothesized that the implant diameters were decreasing due to insufficient material levels in the extrusion chamber. To compensate for this, the extrusion speed was increased, and the speed of the conveyor belt was decreased to lessen the pulling force generated by the conveyor belt (Table 2).

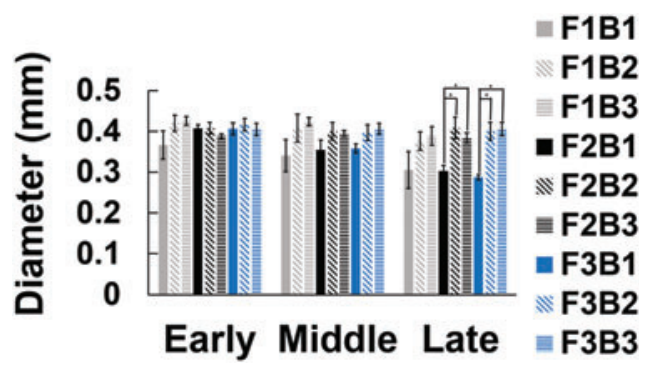

FIG. 1. Dexamethasone-PLGA implant diameters collected from the early, middle, and late phases of implant batch extrusion. Implant batches are listed by formulation $(\mathrm{F} 1=$ fast release, $\mathrm{F} 2=$ intermediate release, and $\mathrm{F} 3=$ slow release) and batch number $(\mathrm{B} 1=$ batch $1, \mathrm{~B} 2=$ batch 2 , and B3 = batch 3). Batch 1 of each formulation used the batch process without manual control of extrusion speed or conveyor belt speed in the late phase. Batches 2 and 3 (B2 and B3) of each formulation are batches with manual control of the above process parameters during the late phase. Error bars represent standard deviation ( $N=10$ or 15 implants). Asterisks indicate statistical difference at $P<0.05$. PLGA, poly (D,Llactide-co-glycolide). Color images are available online.
These process adjustments reduced the decline in diameter and resulted in implants having a more consistent diameter throughout the batch (Fig. 1).

\section{Mechanical properties of implants decreased during extrusion}

Implant mechanical strength and toughness measurements are shown in Fig. 2. In B1 batches, all 3 implant formulations exhibited a decreasing tendency in mechanical properties in the late phase, potentially due to a decline in implant diameter in this late phase. In B2 and B3 batches, all 3 implant formulations tended to exhibit higher mechanical properties in the late phase of extrusion relative to B1, possibly due to generation of implants with more consistent diameters in all phases. Furthermore, when implant mechanical properties were plotted against their diameters for the various batches and formulations, increased diameter correlated with increased mechanical properties (Fig. 3). Thus, changes in diameter appear to influence mechanical properties of implants.

\section{Consistent drug loading was observed throughout the extrusion process for all implant formulations, while F1 formulation showed a higher burst release of dexamethasone}

As shown in Fig. 4, percent dexamethasone loading was not statistically different between early, middle, and late

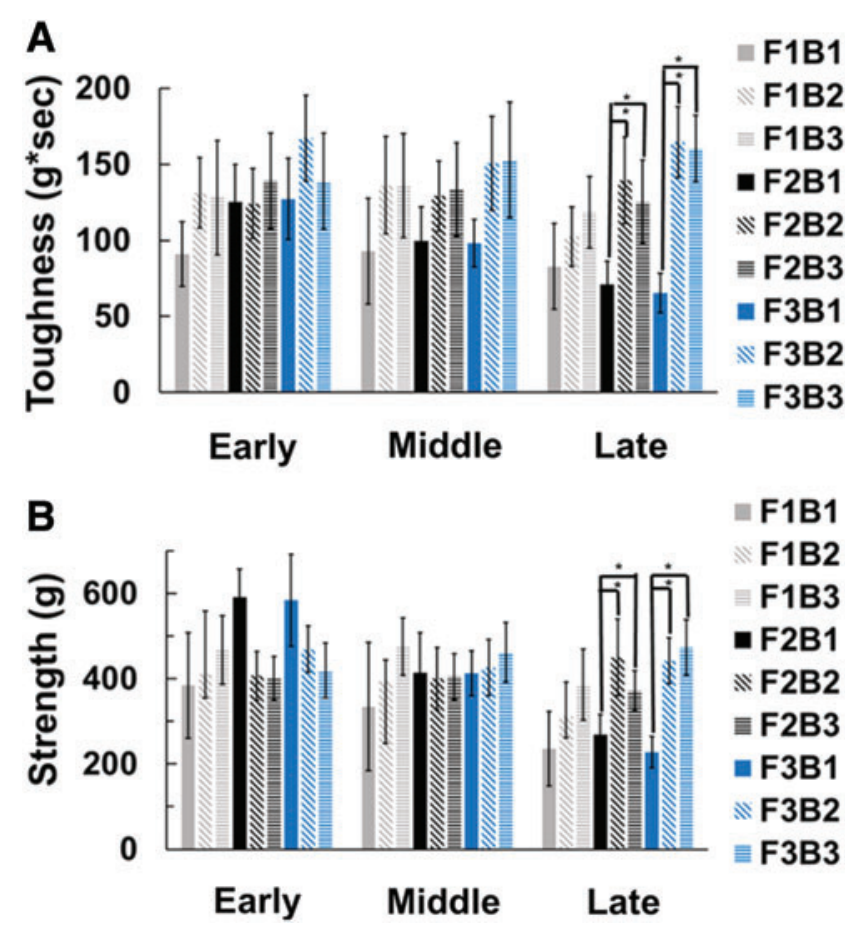

FIG. 2. Toughness and strength (A and $\mathbf{B})$ of dexamethasonePLGA implants during the early, middle, and late phases of the extrusion process. $\mathrm{F} 1=$ fast release, $\mathrm{F} 2=$ intermediate release, and $\mathrm{F} 3=$ slow release. $\mathrm{B} 1=$ batch 1 and $\mathrm{B} 2=$ batch 2. Error bars represent standard deviation $(N=10$ or 15 implants). Asterisks indicate statistical difference at $P<$ 0.05. Color images are available online. 

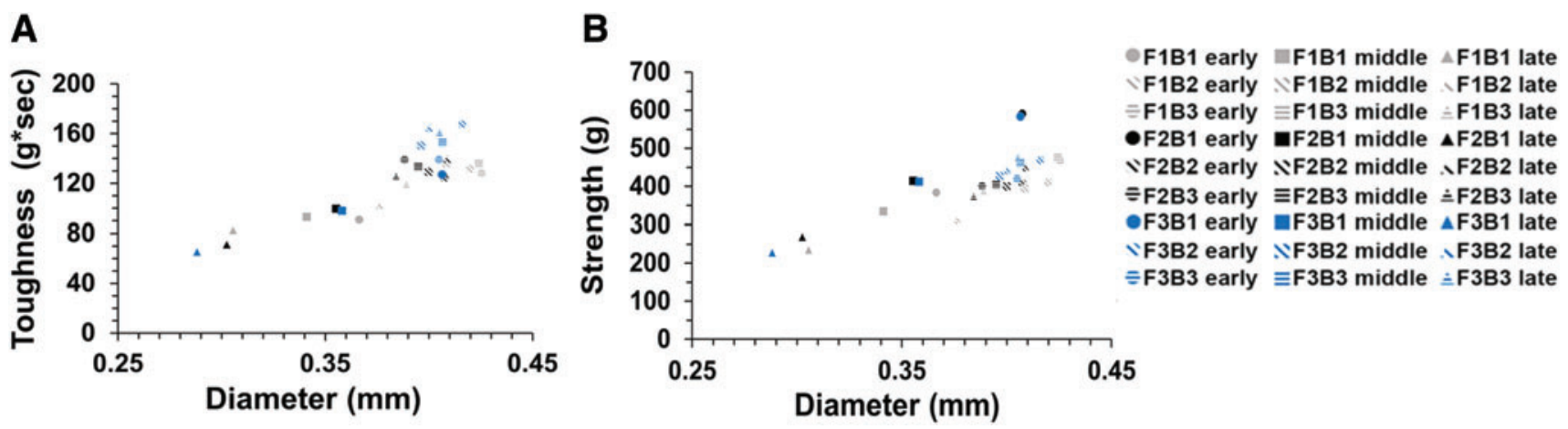

FIG. 3. Correlation of dexamethasone-PLGA implant mechanical properties with implant diameter. Circles represent early extrusion implants, squares represent middle extrusion implants, and triangles represent late-phase extrusion implants. The relationship was linear and explained by the equations, (A) implant toughness in $\mathrm{g} \times \mathrm{s}=624$ (diameter in $\mathrm{mm}$ ) - 117 , $R^{2}=0.73$ and (B) implant strength in $\mathrm{g}=1,785$ (diameter in $\mathrm{mm}$ ) - 277, $R^{2}=0.62$. Color images are available online.

extrusions. Thus, within each batch, the late extrusions tended to show a smaller diameter and weaker mechanical strength, but percent drug loading remained consistent throughout.

Finally, we performed in vitro release experiments to determine if drug release was consistent between batches and within a given batch (Fig. 4). For the F1 (fast release) formulation, the release was very fast, with $40 \%$ or greater release in 1 week, followed by slower release. F2 and F3 formulations released dexamethasone more steadily during the study. For formulations 2 and 3 (Fig. 5), the modified process in the late phase resulted in batches with release profiles similar to those produced with the fixed process. For $\mathrm{F} 1$, multiple release values fell outside of $\mathrm{TL} \pm 15 \%$ for both
FIG. 4. Percent drug loading and cumulative drug release for dexamethasone-PLGA implants from early, middle, and late phases of extrusion. (A) Percent drug loading. Error bars represent standard deviation $(N=3$ or 4$)$. Figures (B-D) Cumulative in vitro drug release. Error bars represent standard deviation $(N=3)$. Color images are available online.

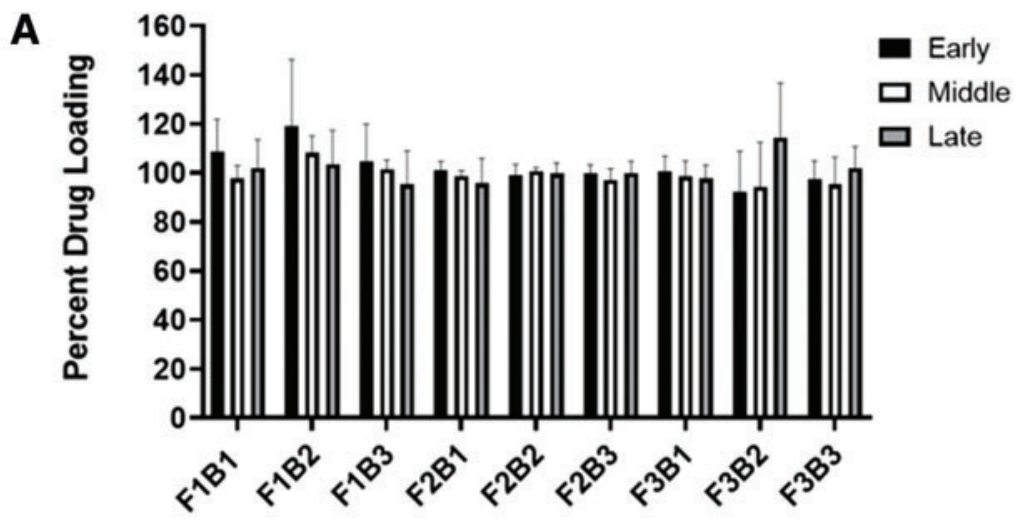

B
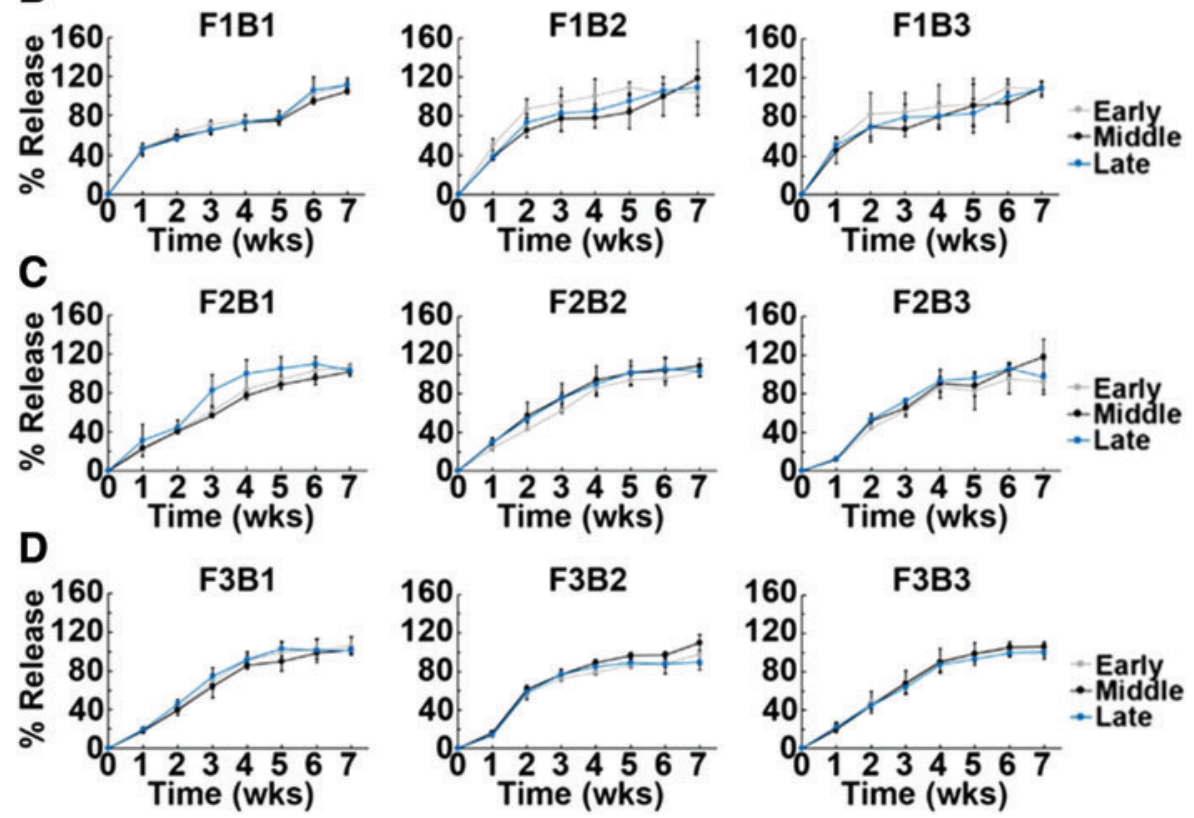

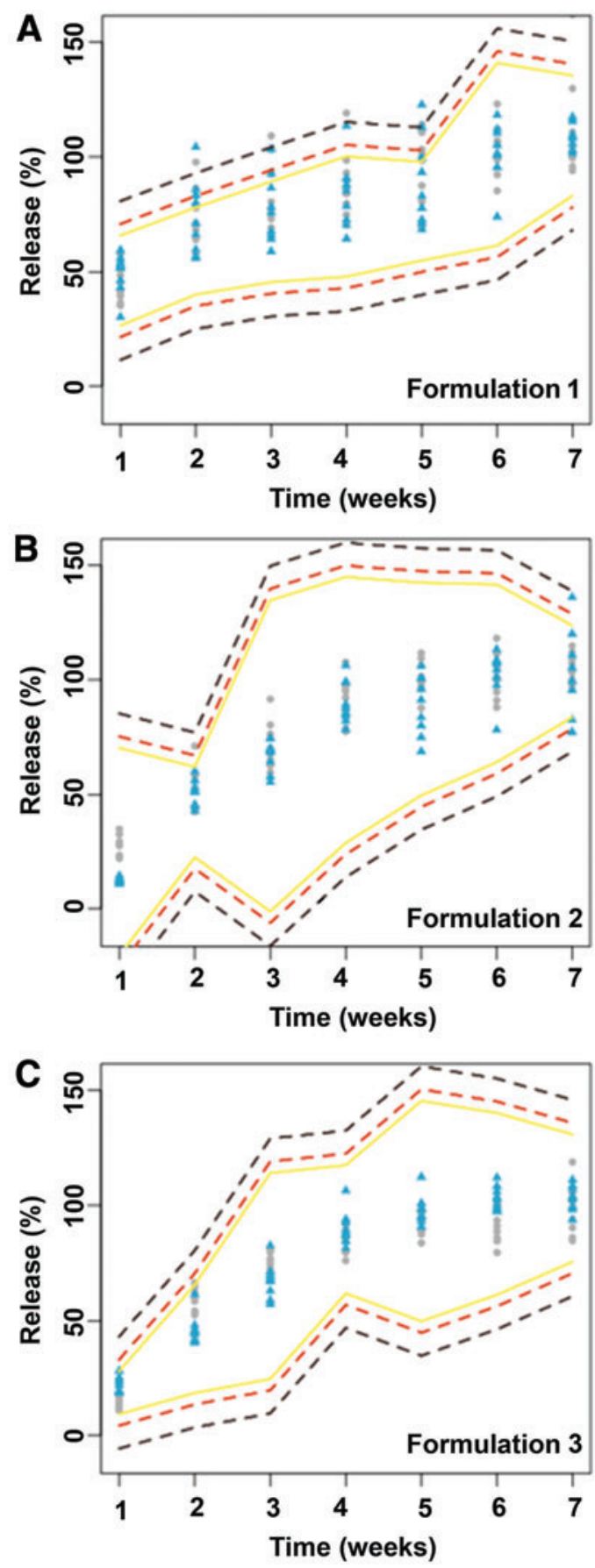

FIG. 5. Similarity of the release profiles of batches produced with and without late-phase process modification for three different compositions of dexamethasone-PLGA implants (A-C). Comparisons of in vitro release profiles for different batches were performed using the modelindependent TL method by Martinez and Zhao. The $99 \%$ TLs were defined with $95 \%$ confidence using batch 1 (fixed process) as the reference (gray line, $n=9$ ). TL $\pm 5 \%$ (gray dashed line) and TL $\pm 15 \%$ (black dashed line) are also plotted. Release values from batch 2 (gray circles, $n=9$ ) and batch 3 (dark gray triangles, $n=9$ ) with late-phase extrusion/conveyor speed modifications are considered to be similar to the batch with the fixed process if all values of the release profile fall within $\mathrm{TL} \pm 15 \%$. TL, tolerance limit. Color images are available online.

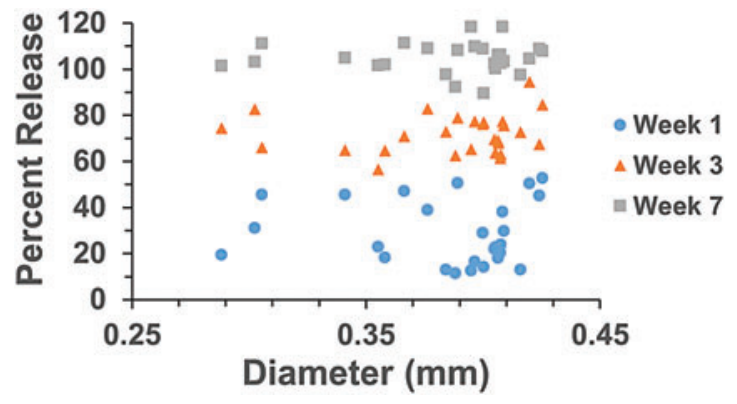

FIG. 6. Correlation of average cumulative percent drug release for F1, F2, and F3 formulations at 1-, 3-, and 7-week time points with the average implant diameters for the respective formulations. No significant relationship was observed. Color images are available online.

batches 2 and 3; therefore, these batches are dissimilar to batch 1 . The release profiles for early, middle, and late phases of a given batch appeared visually similar for a given batch.

Addition of ester PLGA to the implant formulation did result in a slower initial and more consistent drug release; however, increasing this concentration from $16 \%$ to $32 \%$ did not result in significant changes in the release. Furthermore, drug release for various batches and formulations did not correlate with implant diameters in the range used in the present study (Fig. 6).

\section{Discussion}

Drug-loaded, biodegradable polymeric implants allow sustained intravitreal drug delivery in treating intraocular diseases, including uveitis and diabetic macular edema. It is very likely that the implants are made using a batch hot-melt extrusion process for commercial purposes. To ensure reproducible therapy, it is critical that the quality of implants is maintained from one dose to another and between different manufacturing lots. One purpose of this study was to assess the variation in quality and release parameters within and between batches of dexamethasone-loaded PLGA implants. Another purpose of this study was to compare the drug product quality and release parameters for different PLGA compositions. These aspects were tested by assessing implant diameter, strength, toughness, drug loading, and drug release to ensure that implants produced by hot-melt extrusion can withstand the injection procedure without breaking and sustain drug delivery to the back of the eye.

The findings of this study indicated that there was a decline in diameter of the dexamethasone-PLGA implants from batches prepared with 3 different compositions from the beginning to the end of the hot-melt extrusion process. This may be attributed to a great reduction in the volume of the drug-polymer mix in the late phase of extrusion, resulting in thinner extrudate fibers, which were cut into implants. The decrease in implant diameters could be minimized by adjusting the process parameters (increasing the extrusion speed and reducing the conveyor speed beginning at $42 \mathrm{~min}$ during the 60 -min extrusion process). With this modified process, implants exhibited more consistent diameters throughout the process (Fig. 1). A decrease in implant diameters was associated with a decrease in implant strength and toughness (Fig. 2) and there was a moderate linear correlation between implant diameter and 
mechanical properties (Fig. 3). This suggests that implant diameter is a key contributor to the implant mechanical properties. Thus, an increase in implant diameter in the late phases of the modified manufacturing process may have improved the mechanical properties of implants across batches and formulations. Further comparison of implants indicated no significant differences in drug loading, within or between batches, with or without the process modification (Fig. 4).

Fast release implants exhibited greater burst release compared with the intermediate and slow release implants (Fig. 4), which appeared comparable in release profiles and released the drug more steadily over the course of the 7week study. These findings suggest that factors other than polymer degradation might be contributing to drug release. The drug release profiles appear similar across the extrusion processes within a batch for early, middle, and late implants (Fig. 4). Based on the Martinez and Zhao method for comparing drug release profiles, while formulations 2 and 3 did not differ in release profiles with and without process modification during the late phase, formulation 1 exhibited different release profiles with and without process modification (Fig. 5). Thus, drug release from fast release composition appears more sensitive to process changes. Implementation of the Martinez and Zhao method to determine drug release profile similarity in this study is limited by the small sample size ( $n=9$ for each batch analysis vs. $n=36$ or $n=60$ used in the original work). Additionally, implant diameters in the range obtained did not seem to correlate in any way with drug release (Fig. 6). Taken together, these results show that control of extrusion parameters in the late phase of a batch may ensure consistent implant extrusion, thereby producing implants of similar diameter, strength, drug loading, and release during the entire batch.

While our results indicate that adjustment of extrusion and conveyor belt speeds in the late phase of manufacturing may result in a more consistent implant extrusion, this does not represent a feasible strategy to mass produce dexamethasone-PLGA implants. An alternative approach is to design an in-process parameter to monitor the mass extrusion rate and adjust the extrusion and conveyor speeds accordingly. Ideally, a laser micrometer could be used to monitor the diameter of implants being extruded and adjust the extrusion and conveyor speeds in real time; that is, the hot-melt extrusion program could adjust the speeds according to the current diameter being targeted with the die plate.

Given the recent success of dexamethasone implants in the treatment of ocular inflammation, it is important to characterize the implant manufacturing process in a laboratory setting to ensure reproducibility of the process. Dexamethasone implant application is a relatively simple procedure being accomplished in a doctor's office with local anesthesia. ${ }^{27}$ As shown in this study, proper control of the extrusion process results in consistent implant diameter and strength, which is expected to translate to a consistent injection procedure and implant performance. The strength of the Ozurdex implant has been reported to be $\sim 2.75 \mathrm{~N}$ or $280 \mathrm{~g}$. The implant strengths measured in this study are similar or better. ${ }^{28}$

Dexamethasone can be detrimental to corneal endothelial cells above concentrations of $10^{-4}$ to $10^{-3} \mathrm{M}$, causing in- creased apoptosis/necrosis and increased $\mathrm{Na}^{+} \mathrm{K}^{+}$-ATPase activity. ${ }^{29}$ Furthermore, doses of dexamethasone over $1 \mathrm{mg}$ were toxic to the ocular tissues of rabbits. ${ }^{20}$ While implants do not contain these levels of dexamethasone, if not injected or released properly, local levels surrounding ocular tissues could reach a high level. Dexamethasone from PLGA implants has been shown to persist in monkey and rabbit vitreous and retina for up to 6 months. ${ }^{22,23}$ Furthermore, the beneficial effects of dexamethasone have been shown to wear off following complete dissolution of the implant and elimination of the drug. Taken together, these results from the literature show that dexamethasone treatment requires proper control of drug levels across repeated administrations. Better control of the manufacturing process could result in more consistent implant production, thereby ensuring that implant application and performance are reproducible. Quality implants and consistent administration will provide consistent therapeutic and pharmacological effects. In this study, implants maintained a consistent drug loading and release profile in the early, middle, and late phases of extrusion. Process modification generally improved the implant diameter as well as mechanical properties of implants. For 2 of the 3 compositions, the process modification did not affect the release profile, suggesting that the pharmacological effect of the drug would be consistent between different batches or lots.

In conclusion, we could produce dexamethasone-PLGA implants of consistent quality by controlling the parameters of the extrusion process. Hot-melt extrusion is a wellestablished technique to manufacture implants consisting of different materials. However, as the volume of material begins to diminish in the barrel chamber, implant properties begin to vary. Variance in mechanical properties could have potential ramifications during the application of the implant. Similarly, variance in pharmacological properties could result from differences in drug release in the vitreous humor and/or absorption of the drug by the posterior eye tissues. Production of implants with highly consistent mechanical and release properties will aid in the proper treatment of ocular inflammation through sustained dexamethasone delivery to the posterior eye while also minimizing potential side effects of corticosteroid application.

\section{Acknowledgment}

The authors are thankful to Dr. Marilyn Martinez of the US Food and Drug Administration for sharing an example R code for comparing dissolution profiles.

\section{Disclaimer}

The views expressed in this article are those of the authors and should not be considered as FDA's views or policies.

\section{Author Disclosure Statement}

No competing financial interests exist.

\section{Funding Information}

This work was supported by FDA grants, U01 FD004929 and U01 FD004719. The preparation of this article was supported, in part, by the NIH grant, R21EY029887. 


\section{References}

1. Abadia, B., Calvo, P., Ferreras, A., Bartol, F., Verdes, G., and Pablo, L. Clinical applications of dexamethasone for aged eyes. Drugs Aging. 33:639-646, 2016.

2. Herrero-Vanrell, R., Cardillo, J.A., and Kuppermann, B.D. Clinical applications of the sustained-release dexamethasone implant for treatment of macular edema. Clin. Ophthalmol. 5:139-146, 2011.

3. Leopold, I. Nonsteroidal and steroidal anti-inflammatory agents. Surg. Pharmacol. Eye. 83-133, 1985.

4. Ando, N., Sen, H., Berkowitz, B., Wilson, C., and Dejuan, E. Localization and quantification of blood-retinal barrier breakdown in experimental PVR using contrast-enhanced MRI. Invest. Ophthalmol. Vis. Sci. 33:1124-1124, 1992.

5. Matsuda, S., Gomi, F., Oshima, Y., Tohyama, M., and Tano, Y. Vascular endothelial growth factor reduced and connective tissue growth factor induced by triamcinolone in ARPE19 cells under oxidative stress. Invest. Ophthalmol. Vis. Sci. 46:1062-1068, 2005.

6. Sohn, H.J., Han, D.H., Kim, I.T., et al. Changes in aqueous concentrations of various cytokines after intravitreal triamcinolone versus bevacizumab for diabetic macular edema. Am. J. Ophthalmol. 152:686-694, 2011.

7. Penfold, P., Wen, L., Madigan, M., Gillies, M., King, N., and Provis, J. Triamcinolone acetonide modulates permeability and intercellular adhesion molecule-1 (ICAM-1) expression of the ECV304 cell line: implications for macular degeneration. Clin. Exp. Immunol. 121:458-465, 2000.

8. Tamura, H., Miyamoto, K., Kiryu, J., et al. Intravitreal injection of corticosteroid attenuates leukostasis and vascular leakage in experimental diabetic retina. Invest. Ophthalmol. Vis. Sci. 46:1440-1444, 2005.

9. Peyman, G.A., and Herbst, R. Bacterial endophthalmitis. Treatment with intraocular injection of gentamicin and dexamethasone. Arch. Ophthalmol. 91:416-418, 1974.

10. Shah, A., and Branley, M. Use of intravitreal triamcinolone in the management of birdshot retinochoroidopathy associated with cystoid macular oedema: a case study over a three-year period. Clin. Exp. Ophthalmol. 33:442-444, 2005.

11. Karacorlu, M., Karacorlu, S.A., and Ozdemir, H. Intravitreal Triamcinolone Acetonide in Vogt-KoyanagiHarada Syndrome. London, England: SAGE Publications Sage UK; 2006.

12. Pathengay, A. Intravitreal triamcinolone acetonide in serpiginous choroidopathy. Indian J. Ophthalmol. 53, 2005.

13. Karacorlu, M., Ozdemir, H., and Karacorlu, S. Intravitreal triamcinolone acetonide for the treatment of chronic pseudophakic cystoid macular oedema. Acta Ophthalmol. 81:648-652, 2003.

14. Ciardella, A., Klancnik, J., Schiff, W., Barile, G., Langton, $\mathrm{K}$, and Chang, S. Intravitreal triamcinolone for the treatment of refractory diabetic macular oedema with hard exudates: an optical coherence tomography study. $\mathrm{Br}$. J. Ophthalmol. 88:1131-1136, 2004.

15. Massin, P., Audren, F., Haouchine, B., et al. Intravitreal triamcinolone acetonide for diabetic diffuse macular edema1 2: preliminary results of a prospective controlled trial. Ophthalmology. 111:218-224, 2004.

16. Chaudhary, V., Mao, A., Hooper, P.L., and Sheidow, T.G. Triamcinolone acetonide as adjunctive treatment to verteporfin in neovascular age-related macular degeneration: a prospective randomized trial. Ophthalmology. 114:21832189, 2007.
17. Goldfien, A. Adrenocorticosteroids and adrenocortical antagonists. Basic Clin. Pharmacol. Norwalk, CT: Appleton \& Lange, 592-607, 1995.

18. Gilman, A.G. Goodman and Gilman's the Pharmacological Basis of Therapeutics. ( $7^{\text {th }}$ ed.) Macmillan, New York, NY, 1839, 1985.

19. Beer, P.M., Bakri, S.J., Singh, R.J., Liu, W., Peters, G.B., and Miller, M. Intraocular concentration and pharmacokinetics of triamcinolone acetonide after a single intravitreal injection. Ophthalmology. 110:681-686, 2003.

20. Kwak, H.W., and D'Amico, D.J. Evaluation of the retinal toxicity and pharmacokinetics of dexamethasone after intravitreal injection. Arch. Ophthalmol. 110:259-266, 1992.

21. Chan, A., Leung, L.S., and Blumenkranz, M.S. Critical appraisal of the clinical utility of the dexamethasone intravitreal implant (Ozurdex) for the treatment of macular edema related to branch retinal vein occlusion or central retinal vein occlusion. Clin. Ophthalmol. 5:1043-1049, 2011.

22. Chang-Lin, J.E., Attar, M., Acheampong, A.A., et al. Pharmacokinetics and pharmacodynamics of a sustainedrelease dexamethasone intravitreal implant. Invest. Ophthalmol. Vis. Sci. 52:80-86, 2011.

23. Chang-Lin, J.E., Burke, J.A., Peng, Q., et al. Pharmacokinetics of a sustained-release dexamethasone intravitreal implant in vitrectomized and nonvitrectomized eyes. Invest. Ophthalmol. Vis. Sci. 52:4605-4609, 2011.

24. Ferrini, W., and Ambresin, A. Intravitreal dexamethasone implant for the treatment of macular edema after retinal vein occlusion in a clinical setting. Klin. Monbl. Augenheilkd. 230:423-426, 2013.

25. RothenWeinhold, A., Besseghir, K., and Gurny, R. Analysis of the influence of polymer characteristics and core loading on the in vivo release of a somatostatin analogue. Eur. J. Pharm. Sci. 5:303-313, 1997.

26. Martinez, M.N., and Zhao, X.C. A simple approach for comparing the in vitro dissolution profiles of highly variable drug products: a proposal. AAPS J. 20, 2018.

27. Meyer, C.H., Klein, A., Alten, F., et al. Release and velocity of micronized dexamethasone implants with an intravitreal drug delivery system: kinematic analysis with a high-speed camera. Retina. 32:2133-2140, 2012.

28. Lehner, E., Gundel, D., Liebau, A., Plontke, S., and Mader, K. Intracochlear PLGA based implants for dexamethasone release: challenges and solutions. Int. J. Pharm. 1:1-9, 2019.

29. Hatou, S., Yamada, M., Mochizuki, H., Shiraishi, A., Joko, T., and Nishida, T. The effects of dexamethasone on the $\mathrm{Na}, \mathrm{K}-\mathrm{ATPase}$ activity and pump function of corneal endothelial cells. Curr. Eye Res. 34:347-354, 2009.

Received: June 21, 2019

Accepted: March 7, 2020

Address correspondence to:

Dr. Uday B. Kompella

Skaggs School of Pharmacy

and Pharmaceutical Sciences

University of Colorado Anschutz Medical Campus

12850 E. Montview Boulevard, V20-4129

Aurora, CO 80045

E-mail: uday.kompella@cuanschutz.edu 